\title{
Weiteres über Galactosamin.
}

\author{
Von
}

Fr. N. Schulz und Fr. Ditthorn.

(Aus der chemischen Abtheilung des physiologischen Instituts zu Jena.)

(Der Redaction zugegangen am 9. April 1901.)

Bei Untersuchung des Zuckers, der durch stärkere Säuren aus dem Glycoproteid der Eiweissdrüse des Frosches abgespalten wird, konnten wir Befunde erheben, aus denen wir schlossen, dass es sich um ein Galactosamin handele. ${ }^{1}$ )

$\mathrm{Da}$ die Reindarstellung ausreichender Mengen dieses Zuckers auf grosse, bisher nicht überwundene Schwierigkeiten stiess, so versuchten wir zunächst auf indirektem Wege weiter zu kommen, durch den Versuch, auf andere Weise Galactosamin zu gewinnen.

Es war uns seiner Zeit entgangen, dass der Name Galactosamin eigentlich schon anderweitig vergeben ist. Lobry de Bruyn und van Leent haben unter diesem Namen einen Körper beschrieben, der durch Einwirkung von $\mathrm{NH}_{3}$ in methylalkoholischer Lösung auf Galactose entsteht, und dem die $\mathrm{Zu}$ sammensetzung $\mathrm{C}_{6} \mathrm{H}_{13} \mathrm{NO}_{5}$ zugeschrieben wird. ${ }^{2}$ ) Ausser einem Galactosamin sind noch Ammoniakverbindungen mehrerer anderer Zucker in analoger Weise von den Verfassern her-

1) Fr. N. Schulz und F. Ditthorn, Galactosamin, ein neuer Amidozucker, als Spaltungsprodukt des Glycoproteids der Eiweissdrüse des Frosches. Zeitschr. f. physiol. Chemie, Bd. XXIX, 1900, S. 373.

2) a. Fr. H. van Leent, Ueber Milchzucker, Galactose und Maltose und ihre Ammoniakverbindungen». Inaug.-Diss., Basel, 1894.

b. C. A. Lobry de Bruyn et F. H. Leent, Dérivés ammoniaux de quelques sucres. Recueil des Traveaux chimiques des Pays-Bas. 1895. Tome XIV, S. $140 \mathrm{ff}$. 
gestellt worden. Alle diese Ammoniakverbindungen von Kohlehydraten unterscheiden sich von dem Glycosamin durch die lockere Bindung des Ammoniaks. Schon beim Stehen der wässerigen Lösung sowie bei Berührung der festen Substanz mit Luft wird $\mathrm{NH}_{3}$ abgespalten. Durch verdünnte Säuren (auch organische, z. B. Oxalsäure) wird das locker gebundene $\mathrm{NH}_{3}$ quantitativ frei gemacht. Es gelingt demnach nicht, Salze dieser Ammoniakzucker mit Säuren darzustellen. Umgekehrt ist das Glycosamin nur unter besonderen Vorsichtsmassregeln als freie Base zu erhalten, während es mit Säuren prächtig krystallisirende Salzverbindungen eingeht und dementsprechend nur sehr schwer $\mathrm{NH}_{3}$ abspaltet.

In mancher Beziehung in der Mitte zwischen diesen beiden Gruppen von Ammoniakverbindungen der Kohlehydrate stehen die von Emil Fischer ${ }^{1}$ ) auf anderem Wege dargestellten Amidozucker, das Isoglucosamin und das Akrosamin, beide durch Reduction des betreffenden Osazons mit Zink und Eisessig gewonnen. Diese letzteren sind als freie Basen bisher nicht dargestellt; sie enthalten das Ammoniak in ziemlich fester Bindung, so dass es gelingt, Salze mit organischen Säuren (Acetat und Oxalat des Isoglycosamins, Oxalat des Akrosamins) herzustellen. Beim Behandeln mit Mineralsäuren tritt dagegen eine Abspaltung von $\mathrm{NH}_{3}$ ein, und es ist bisher nicht gelungen, Verbindungen mit Mineralsäuren darzustellen.

De Bruyn und van Leent gaben selbst an, dass ihre Ammoniakderivate sich in ihrer Constitution von den drei anderen damals bekannten Osaminen (Glycosamin, Isoglycosamin, Akrosamin) unterscheiden (S. $142 \mathrm{Anm}$.). Es dürfte daher wohl zweckmässig sein, die Bezeichnung Osamine für solche Zucker zu reserviren, die durch die festere Bindung des $\mathrm{NH}_{3}$ (Salzbildung mit Säuren) als zu einer Gruppe gehörend sich documentiren; zu diesen würde auch das von uns vermuthete Galactosamin zu zählen sein.

1) a. Emil Fischer, Ueber Isoglucosamin. Chem. Ber., Bd. 19, 1886, S. 1920.

b. E. Fischer und J. Tafel, Synthetische Versuche in der Zuckergruppe. Chem. Ber., Bd. 20, 1887, S. 2566-2575. 
Um ein Vergleichsobject für den aus dem Glycoproteid der Eiweissdrüse des Frosches darstellbaren Zucker zu gewinnen, gingen wir in ganz analoger Weise vom Galactosazon aus, wie Emil Fischer aus Glucosazon und Akrosazon das Isoglucosamin und das Akrosamin darstellte, $\left.{ }^{1}\right)$ in der Hoffnung, so ein Galactosamin zu erhalten.

$100 \mathrm{~g}$ Galactose (Kahlbaum), in $1 \mathrm{l}$. Wasser gelöst, wurden mit. $100 \mathrm{~g}$ Phenylhydrazin und $100 \mathrm{~g}$. Essigsäure mehrmals längere Zeit gekocht und die beim Erkalten abgeschiedenen Krystalle jedesmal abfiltrirt, mit Wasser gewaschen und auf Thonplatten an der Luft getrocknet. Die Ausbeute betrug in mehreren Darstellungen je $50 \mathrm{~g}$ Galactosazon.

$50 \mathrm{~g}$ Galactosazon, mit $100 \mathrm{ccm}$. Wasser und $300 \mathrm{ccm}$. Alkohol absolutus verrieben, unter allmählichem Zusatz von $125 \mathrm{~g}$ Zinkstaub und $50 \mathrm{~g}$ Eisessig bei $50^{\circ}$ reducirt. Nachdem die sichtbaren gelben Osazonpartikel vollständig verschwunden, wurde abfiltrirt. Das Filtrat wurde. mit $\mathrm{SH}_{2}$ vom Zink befreit. Diese Procedur erfordert Geduld; das sehr voluminöse $\mathrm{ZnS}$ musste mehrmals abfiltrirt und zur Vermeidung von Verlusten mit Alkohol (1:3 wie oben) ausgewaschen werden. Trotzdem war dieses Entzinken sicher mit bedeutenden Verlusten verbunden. Das völlig zinkfreie Filtrat wurde bei $50^{\circ}$ unter möglichst vermindertem. Druck zum Syrup eingedampft und dann mit reichlich Alkohol und viel Aether gefällt. Der abgeschiedene Syrup zeigte keine Neigung, spontan krystallinisch $\mathrm{zu}$ werden. Nach mehrtägigem Stehen wurde von dem zähen Syrup abdecantirt; der Syrup wurde in wenig Wasser gelöst (wobei er sich fast völlig auflöste) und filtrirt.

Das Filtrat wurde mit dem 20-30 fachen Volumen Alkohol absolutus versetzt. Hierdurch wurde eine geringe Trübung, aber keine nennenswerthe Fällung erzielt.

Das Galactosamin wird also, falls es bei der Reduction des Galactosazons sich bildete, als Acetat aus wässeriger Lösung nicht gefällt, im Gegensatz zum Isoglucosaminacetat. Aehnlich verhält sich nach E. Fischer das Akrosamin, dessen Acetat auch nicht krystallisirt. Da das Akrosamin dagegen ein krystallisirendes Oxalat bildet, und auch das Isoglucosamin-

1) So weit wir sehen, ist (ausser einem nicht weiter verfolgten Versuch mit Lactosazon von Emil Fischer) dies Verfahren nicht anderweitig zur Darstellung von Amidozuckern benutzt worden, obgleich schon Fischer ausdrücklich auf die Bedeutung derartiger.Verbindungen für den Physiologen hinwies und die Hoffnung aussprach, dass auf diesem Wege eine grössere Anzahl von Ammoniakderivaten der Zuckerarten zu gewinnen sei. 
oxalat durch Alkohol leichter gefällt wird, wie das Isoglucosaminacetat, so wurde die wässerig alkoholische Lösung des vermutheten Galactosaminacetats mit alkoholischer Oxalsäure versetzt. Hierdurch liess sich eine reichliche, voluminöse, schön krystallinische Fällung erzielen. Dieselbe wurde abfiltrirt, in wenig Wasser gelöst und nochmals mit Alkohol gefällt. Hierbei zeigte es sich, dass zwei verschieden leicht fällbare Substanzen in der Lösung vorhanden waren.

Die leichter fällbare Substanz, durch mehrfaches Fractioniren mit Alkohol soweit gereinigt, dass sie Fehling'sche Lösung nicht mehr reducirte, erwies sich durch ihre Eigenschaften sowie durch Elementaranalyse als Ammoniumoxalat. Das Ammoniak bildet sich in reichlichen Mengen bei der Reduction des Osazons, die sich nach der Gleichung vollzieht.

$$
\mathrm{C}_{18} \mathrm{H}_{22} \mathrm{~N}_{4} \mathrm{O}_{4}+\mathrm{H}_{8} \mathrm{O}+6 \mathrm{H}=\mathrm{C}_{6} \mathrm{H}_{18} \mathrm{NO}_{5}+\mathrm{NH}_{3}+2 \mathrm{C}_{6} \mathrm{H}_{5} \mathrm{NH}_{2}
$$

Der schwer fällbare Theil enthielt den gesuchten Zucker. Der Versuch, denselben durch Entfernen der ersten Alkoholfällungen, die bei mehrfachem Lösen in Wasser und Ausfällen mit Alkohol erhalten wurden, von anhaftendem Ammonoxalat völlig zu befreien, misslang, wie die nachfolgenden Analysen zeigen.

Eine nicht zu verdünnte Lösung von Ammonoxalat wird schon durch wenig Alkohol gefällt (das Gleiche bis doppelte Volumen). Die Lösung des Zuckers konnte so weit von Ammoniumoxalat befreit werden, dass eine sehr concentrirte Lösung erst durch das 10-20 fache Volumen Alkohol gefällt wurde.

Wir erhielten so ein Präparat, welches rein krystallinisch war und sich durch Waschen mit Alkohol und Aether und Trocknen auf Thonplatte leicht als feines leicht gelbliches Krystallpulver gewinnen liess. Auf dieses Pulver beziehen sich die nachfolgenden Angaben.

1. Reductionsvermögen. $0,0477 \mathrm{~g}$ Galactosaminoxalat reducirten $6,4 \mathrm{ccm}$. Fehling'scher Lösung, entprechend $0,032 \mathrm{~g}$ Traubenzucker.

$0,0477 \mathrm{~g}$ Galactosaminoxalat $\left(\mathrm{C}_{6} \mathrm{H}_{13} \mathrm{NO}_{5} \cdot \mathrm{C}_{2} \mathrm{O}_{4} \mathrm{H}_{2}\right)$ wären äquimolekular mit $0,032 \mathrm{~g}$ Traubenzucker.

$0,0477 \mathrm{~g}$ Galactosaminoxalat $\left(\mathrm{C}_{6} \mathrm{H}_{13} \mathrm{NO}_{5}\right)_{2} \cdot \mathrm{C}_{2} \mathrm{O}_{4} \mathrm{H}_{2}$ wären äquimolekular mit $0,0383 \mathrm{~g}$ Traubenzucker. 
Da das Präparat, wie die Elementaranalyse ergab, noch stärker mit Ammonoxalat verunreinigt war (ca. 25\%), so ist das Reductionsvermögen also etwas grösser wie das des Traubenzuckers.

2. Elementare Zusammensetzung.

\begin{tabular}{cc|c|c|c|c|c}
\hline & \multicolumn{3}{c|}{ Gefunden } & \multicolumn{3}{|c}{ Berechnet für } \\
& $\mathrm{I}$ & $\mathrm{II}$ & Mittel & $\mathrm{C}_{6} \mathrm{H}_{13} \mathrm{~B}_{5} \cdot \mathrm{C}_{2} \mathrm{O}_{4} \mathrm{H}_{2}$ & $\mathrm{C}_{2} \mathrm{O}_{4}\left(\mathrm{BH}_{4}\right)_{2}$ & $\left(\mathrm{C}_{6} \mathrm{~B}_{13} \mathrm{~J}_{5}\right)_{2} \mathrm{C}_{2} \mathrm{O}_{4} \mathrm{H}_{2}$ \\
\hline $\mathrm{C}$ & 33,19 & 33,02 & 33,11 & 35,68 & 16,90 & 37,50 \\
$\mathrm{H}$ & 6,0 & 6,13 & 6,06 & 5,57 & 7,0 & 6,25 \\
$\mathrm{~N}$ & 9,7 & 9,74 & 9,72 & 5,20 & 19,72 & 6,25
\end{tabular}

Die gefundenen Werthe würden annähernd stimmen für 25\% Ammonoxalat $+75 \%$ Galactosaminoxalat, und zwar besser für $\left(\mathrm{C}_{6} \mathrm{H}_{13} \mathrm{NO}_{5}\right)_{2} \mathrm{C}_{2} \mathrm{O}_{4} \mathrm{H}_{2}$. Dem Isoglucosaminoxalat kommt die Formel $\mathrm{C}_{6} \mathrm{H}_{13} \mathrm{NO}_{5} \cdot \mathrm{C}_{2} \mathrm{O}_{4} \mathrm{H}_{2} \mathrm{zu}$; dem Akrosaminoxalat die Formel $\left(\mathrm{C}_{6} \mathrm{H}_{13} \mathrm{NO}_{5}\right)_{2} \mathrm{C}_{2} \mathrm{O}_{4} \mathrm{H}_{2}$.

3. Optische Activität. Die Substanz war optisch inactiv (eine 10\% ige Lösung zeigte keine mit einem Saccharimeter nachweisbare Drehung). Die Salze des Isoglycosamins sind stark linksdrehend; das 0xalat des Akrosamins ist optisch inactiv.

4. Bei Oxydation mit Salpetersäure bildet sich Schleimsäure, die durch die typischen, in kaltem Wasser sehr schwer löslichen Krystalle nachgewiesen werden konnte.

5. Die wässerige Lösung färbt sich beim Erwärmen mit Natronlauge erst gelb, dann braun (unter $\mathrm{NH}_{3}$-Entwickelung). Beim Ansäuren trat intensiver Geruch nach Karamel auf. Auch beim einfachen Veraschen der Substanz machte sich dieser Geruch bemerkbar.

6. Mit Phenylhydrazin wird sehr leicht Osazon regenerirt. Schon in der Kälte wird nach einigem Stehen (24 Stunden) einer Zuckerlösung mit Essigsäure und Phenylhydrazin in reichlicher Menge Osazon abgeschieden. Nach kurzem Kochen tritt Osazon reichlicher auf. Schmelzpunkt nach einmaligem Umkrystallisiren $163^{\circ} \mathrm{C}$. Ein weiteres Reinigen musste aus Mangel an Material unterbleiben. 
Aus den vorliegenden Daten geht hervor, dass bei der Reduction des Galactosazons ein Galactosamin entsteht, welches mit Oxalsäure ein aus wässeriger Lösung durch Alkohol tällbares Oxalat bildet.

Die Ausbeute war sehr schlecht; aus $150 \mathrm{~g}$ Galactosazon erhielten wir nur 2,5 $\mathrm{g}$ analysenfähiges Galactosamin (noch mit Ammonoxalat verunreinigt, s. oben). Die verschiedenen durch Alkohol unvollständig gefällten Mutterlaugen lieferten beim Verdunsten noch mehrere Gramm (ca. $3 \mathrm{~g}$ ) einer Fehling'sche Lösung sehr intensiv reducirenden Masse. Dieselbe war stark hygroskopisch, wurde an der Luft sofort syrupös und liess sich nicht in analysenfähige Form bringen.

Die Menge des als leichter durch Alkohol fällbar, durch Fractioniren abgetrennten Ammonoxalats betrug $1,2 \mathrm{~g}$; dasselbe war nahezu rein.

Berechnet für $\mathrm{C}_{8} \mathrm{O}_{4}\left(\mathrm{NH}_{4}\right)_{2}+\mathrm{H}_{8} \mathrm{O}=\mathrm{C}, 16,90, \mathrm{H} 7,0, \mathrm{~N} 19,72,036,3$,

Gefunden $\mathrm{C}$ 16,59, H 6,84, N 20,5, 0 56,07.

Zum Vergleich mit dem früher aus dem Froschglycoproteid dargestellten Zucker wurde $1 \mathrm{~g}$ des möglichst gereinigten Galactosaminoxalates mit $5 \mathrm{ccm}$. Wasser gelöst, sodann mit $5 \mathrm{ecm} .10 \%$ iger $\mathrm{HCl}$ und einem grossen Ueberschuss von Alkohol versetzt. Auch durch 20-30fache Menge Alkohol trat keine Fällung ein; erst durch Zusatz von reichlichen Mengen Aether konnte eine solche erzielt werden. Die aus Krystallen bestehende ausgeschiedene Masse war so hygroskopisch, dass sie nur in Form eines zähen Syrups erhalten werden konnte, in welchen sie an der Luft sofort zerfloss. Der Syrup enthielt reichlich Chlor, reducirte Fehling'sche Lösung intensiv. Beim Behandeln mit absolutem Alkohol ging ein Theil der reducirenden Substanz in Lösung, ein Theil blieb ungelöst. Von diesem ungelösten Theil wurde, nachdem derselbe durch Stehen an der Luft wieder Wasser angezogen, von Neuem ein Theil durch Alkohol gelöst, so dass nach und nach fast die ganze Substanz in eine starke Alkohollösung übergeführt werden konnte.

Wir haben also ganz ähnliche Verhältnisse vor uns, wịe sie seinerzeit bei dem aus dem Glycoproteid des Frosches gewonnenen Zucker zur Beobachtung kamen. Damals wurde 
die Alkohollöslichkeit der HCl-Verbindung des Amidozuckers als Unterscheidungsmerkmal gegenüber dem Glycosamin hervorgehoben. Wahrscheinlich ist diese Angabe dahin zu modificiren, dass nur unreine, hygroskopische Präparate eine derartige Alkohollöslichkeit besitzen. $\mathrm{Ob}$ es gelingen wird, krystallinische Präparate des salzsauern Galactosamins zu gewinnen, und ob diese in Alkohol unlöslich sind, mag die Zukunft lehren; auf jeden Fall ist das Verhalten des salzsauren Glycosamins gegenüber Alkohol in typischer Weise verschieden von dem des Galactosaminchlorids.

Unsere jetzigen Erfahrungen sind dazu geeignet, die früher ausgesprochene Vermutung, dass ein Galactosamin ein Paarling des Glycoproteids der Froscheiweissdrüse sei, wesentlich zu unterstützen.

Die nächsten Aufgaben werden sein, erstens aus dem Galactosazon wirklich reines Galactosamin darzustellen. Zweitens ist $\mathrm{zu}$ versuchen, ob der aus den untersuchten Glycoproteiden abspaltbare Zucker in Gestalt des Oxalats, die für die Abscheidung offenbar die günstigsten Bedingungen darbietet, in reiner Form $\mathrm{zu}$ gewinnen ist. 